\title{
Sliding-induced Microstructure of Cold-Sprayed Copper Coating Observed by Electron Channeling Contrast Imaging
}

\author{
Yinyin Zhang ${ }^{1}$, Nicolas Brodusch ${ }^{1}$, J. Michael Shockley ${ }^{1}$, Raynald Gauvin ${ }^{1}$, and Richard R. Chromik ${ }^{1}$ \\ ${ }^{1 .}$ Department of Mining and Materials Engineering, McGill University, Montreal, Quebec, Canada.
}

Cold gas dynamic spray is a coating process for fabrication of materials that are sensitive to heat and oxidation such as copper [1]. In cold spray, the particles are accelerated to supersonic velocities typically between $500-1200 \mathrm{~m} / \mathrm{s}$ [1]. Upon high speed impact, the cold sprayed particles undergo extensive plastic deformation at very high strain-rates (up to $10^{9} \mathrm{~s}^{-1}$ near impact interfaces), under which conditions complex microstructures are generated such as high density dislocation walls, deformation twins, elongated and equi-axed grains and sub-grains [2-3].

When cold sprayed coatings are subjected to sliding wear, an already complex microstructure is subjected to stress and will further evolve with grain refinement, work hardening, and crack formation [4]. Tribological behaviour of cold-sprayed materials, including the microstructural evolution, could be different from that of traditional bulk materials. In this work, we use the Electron Channeling Contrast Imaging (ECCI) technique in the scanning electron microscope (SEM) to study the microstructure of cold-sprayed $\mathrm{Cu}$ after sliding wear testing.

The sliding wear tests were conducted by a custom-built reciprocating tribometer [5] in ambient atmosphere using an alumina ball as counterface. The initial average Hertzian stress was $687 \mathrm{MPa}$ and the sliding distance $24 \mathrm{~m}$. Electron channeling contrast images were captured at a beam voltage of 15 $\mathrm{kV}$ and a sample tilt over a range of a few degrees using the photodiode semi-conductor in-chamber and in-lens BSE detectors.

ECCI was used to examine sliding-induced microstructures of cold-sprayed coating. For comparison, the as-sprayed coating, shown in Figure 1a, consisted of regions of refined recrystallized grains and relatively coarse grains. At the surface of the wear track, as shown in Figure 1b, a mechanically mixed layer (MML) of nanograins was observed. In the subsurface regions, well-defined equiaxed grains/subgrains were found and studied using different diffraction conditions. At $0^{\circ}$ tilt, as shown in Figure $1 \mathrm{c}$, grains/sub-grains and dislocations have the best contrast within the dark grains, which are those closer to Bragg condition. While tilting the sample to $+2^{\circ}$, as seen in Figure 1d, some grain/sub-grain boundaries and dislocations that were not visible in Figure 1c were observed with good contrast in the newly darkened grains (near Bragg condition). Based on the above observations, sliding wear creates a distinct MML, but subsurface deformations are more difficult to interpret. Many of the highly refined recrystallized regions of the starting microstructures (Figure 1a) are no longer evident in the subsurface of the worn microstructure (Figure 1b). However, coarse structures in Figure 1a, where abrupt change in contrast in the band contour is assumed to be related to plastic deformation and induced dislocations that were probably tangled [6], appeared to undergo microstructural evolution to a less stressed state over sliding.

\section{References}

[1] V.K.A. Papyrin et al in "Cold Spray Technology", (Elsevier, Amsterdam) pp. 33-115.

[2] H. Assadi et al, Acta Materiala 51 (2003), pp. 4379-4394. 
[3] C. Borchers et al, J.Appl.Phys 96 (2004), pp. 4288-4292.

[4] Y. Berthier in "Wear-Materials, Mechanisms and Practice", (John Wiley and Sons, Ltd.), pp. 291316.

[5] H.W. Strauss et al, Wear 272 (2011), pp. 133-148.

[6] B.C. Ng et al, Mater. Sei. Eng A 239 (1997), pp.150-156.
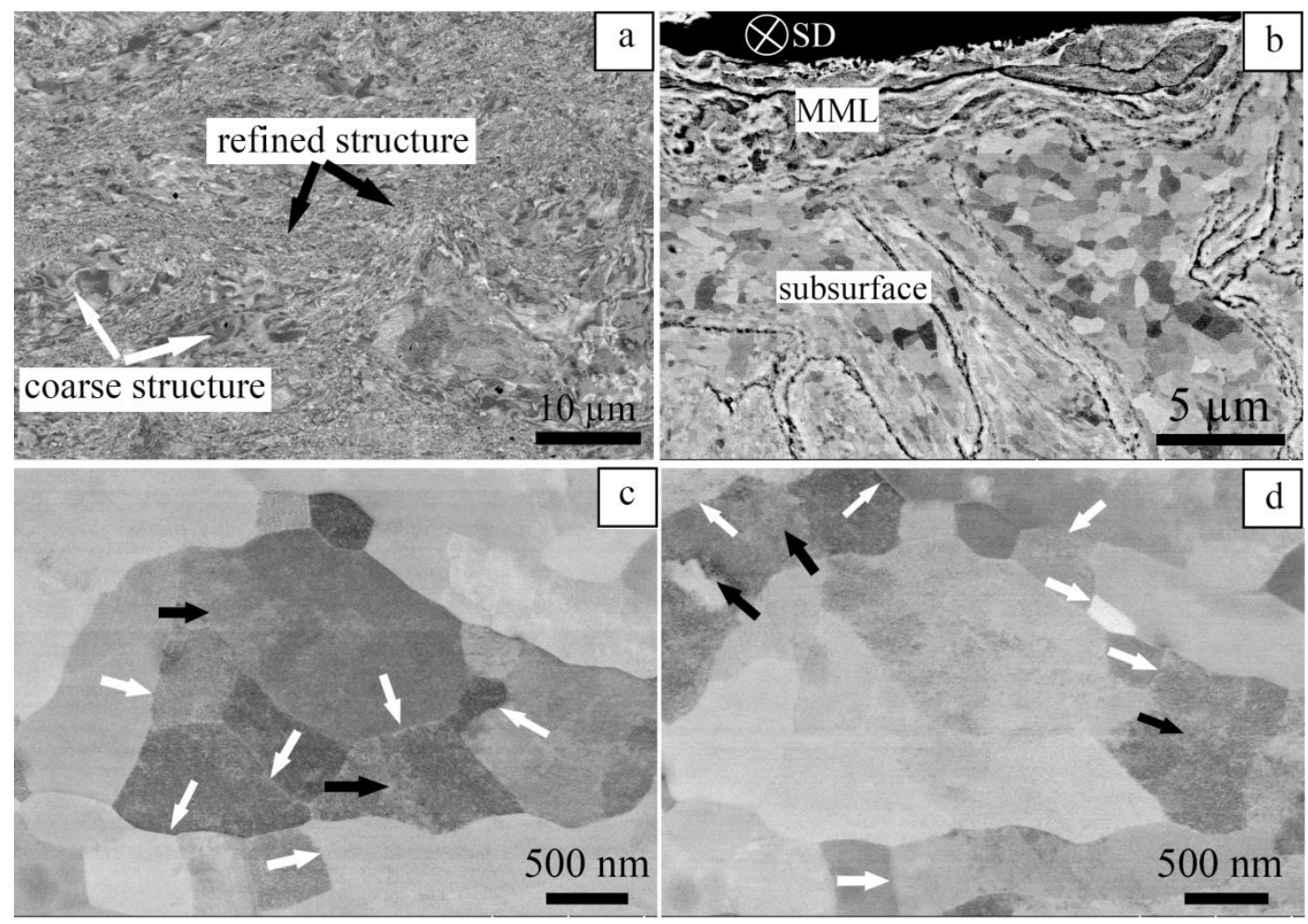

Figure 1: Electron channeling contrast images of cross-section of cold-sprayed $\mathrm{Cu}$ coating before and after sliding wear test, where sliding direction was going into the screen and denoted as SD. (a) shows inhomogeneous microstructure including refined recrystallized regions as well as coarse grain regions, (b) shows highly deformed structures within mechanically mixed layer (MML). From the subsurface, (c) and (d) are presenting lattice defects with sample tilt of $0^{\circ}$ and $2^{\circ}$, respectively, where grain/subgrain boundaries are denoted as white arrows while dislocations black arrows. 